\title{
Capacitação profissional e sua articulação na assistência de enfermagem à criança
}

\section{com câncer}

\author{
Professional training and its articulation in the nursing care to the child with cancer \\ Capacitación profesional y su articulación en la asistencia de enfermería al niño con cáncer
}

Recebido: 26/04/2021 | Revisado: 04/05/2021 | Aceito: 06/05/2021 | Publicado: 20/05/2021

\author{
Ana Paula Batista dos Santos \\ ORCID: https://orcid.org/0000-0002-4295-6903 \\ Universidade Federal de Sergipe, Brasil \\ E-mail: paulinha7b7@gmail.com \\ Rute Nascimento Oliveira Santos \\ ORCID: https://orcid.org/0000-0002-5271-6436 \\ Universidade Federal de Sergipe, Brasil \\ E-mail: rutenascimento0310@gmail.com \\ Queila Samara dos Santos Farias \\ ORCID: https://orcid.org/0000-0002-4686-4539 \\ Universidade Tiradentes, Brasil \\ E-mail: queilasamara20@gmail.com \\ Jéssica Lorrane Barreto Silva Santos \\ ORCID: https://orcid.org/0000-0001-9837-2539 \\ Universidade Tiradentes, Brasil \\ E-mail: jesylorrane@hotmail.com \\ Manuela de Carvalho Vieira Martins \\ ORCID: https://orcid.org/0000-0003-1222-5955 \\ Universidade Tiradentes, Brasil \\ E-mail: manuela.cvm@ hotmail.com \\ Fernanda Costa Martins Gallotti \\ ORCID: https://orcid.org/0000-0002-9063-1273 \\ Universidade Tiradentes, Brasil \\ E-mail: fernanda.gallotti@souunit.com.br
}

\begin{abstract}
Resumo
$\mathrm{O}$ presente trabalho objetiva identificar a frequência de capacitação da equipe de enfermagem de uma unidade oncológica pediátrica e a articulação dos conhecimentos adquiridos com a assistência prestada. A fim de atingir o objetivo proposto e tendo como caminho metodológico o estudo transversal, de cunho descritivo e abordagem quantitativa. A pesquisa foi realizada em um Hospital de referência de Sergipe, onde foram abordados 63 profissionais da equipe de enfermagem. A coleta foi realizada de forma individual entre os meses de março e abril de 2017. Os dados coletados foram organizados em um banco de dados e analisados através da técnica de análise de dados. Os resultados demonstraram uma deficiência das capacitações na área de oncologia pediátrica, mais da metade dos profissionais não obtiveram nenhum tipo de conhecimento voltado para essa temática no seu processo de formação, além da baixa oferta das capacitações por parte do setor atual com uma frequência de apenas 12,7\% e pouca procura por parte dos profissionais, mesmo com uma concepção dos benefícios das capacitações. Por fim, evidenciou-se um déficit na frequência das capacitações, direcionado à oncologia pediátrica, da formação à prática profissional, apresentando-se como grande obstáculo para uma assistência qualificada.
\end{abstract}

Palavras-chave: Capacitação profissional; Enfermagem; Oncologia; Pediatria.

\begin{abstract}
The present work aims to identify the frequency of training of the nursing staff of a pediatric oncology unit and the articulation of the knowledge acquired with the assistance provided. In order to achieve the proposed objective and having the cross-sectional study as a methodological approach, of a descriptive nature and quantitative approach. The research was carried out in a reference hospital in Sergipe, where 63 professionals from the nursing team were approached. The collection was carried out individually between the months of March and April 2017. The collected data were organized in a database and analyzed using the data analysis technique. The results showed a deficiency in training in the area of pediatric oncology, more than half of the professionals did not obtain any type of knowledge focused on this theme in their training process, in addition to the low supply of training by the current sector with a frequency of only $12.7 \%$ and little demand from professionals, even with a conception of the benefits of training. Finally, there was a deficit in the frequency of training, directed to pediatric oncology, from training to professional practice, presenting itself as a major obstacle to qualified assistance.
\end{abstract}

Keywords: Professional training; Nursing; Oncology; Pediatrics. 


\begin{abstract}
Resumen
El presente trabajo tiene como objetivo identificar la frecuencia de formación del equipo de enfermería de una unidad de oncología pediátrica y la articulación de los conocimientos adquiridos con la asistencia brindada. Para lograr el objetivo propuesto y teniendo como camino metodológico el estudio transversal, de carácter descriptivo y enfoque cuantitativo. La investigación se llevó a cabo en un hospital de referencia de Sergipe, donde se acercó a 63 profesionales del equipo de enfermería. La recolección se realizó de manera individual entre los meses de marzo y abril de 2017. Los datos recolectados se organizaron en una base de datos y se analizaron mediante la técnica de análisis de datos. Los resultados evidenciaron una deficiencia en la formación en el área de oncología pediátrica, más de la mitad de los profesionales no obtuvieron ningún tipo de conocimiento enfocado en esta temática en su proceso de formación, además de la baja oferta de formación por parte del sector actual con una frecuencia de tan solo el $12,7 \%$ y poca demanda por parte de los profesionales, incluso con una concepción de los beneficios de la formación. Finalmente, hubo un déficit en la frecuencia de la formación, dirigida a la oncología pediátrica, desde la formación hasta la práctica profesional, presentándose como un gran obstáculo para la asistencia calificada.
\end{abstract}

Palabras clave: Capacitación professional; Enfermería; Oncología; Pediatría.

\title{
1. Introdução
}

Os cânceres em crianças e adolescentes com faixa etária entre 0 a 19 anos são em grande parte o resultado de alterações no DNA das células, acometendo muitas vezes antes do nascimento e, configuram-se como um relevante problema para saúde pública tanto em países desenvolvidos quanto em países que encontram-se em desenvolvimento (Feliciano e Pombo-de-Oliveira, 2018). Em oposição aos adultos, o câncer infantil não está relacionado ao estilo de vida e a fatores ambientais e, tendem a responder melhor aos tratamentos, uma vez que normalmente os organismos desses jovens não apresentam muitos problemas de saúde por causa da sua pouca idade (Instituto Oncoguia, 2020).

Na infância, o câncer é uma doença de alta letalidade e possui diversas apresentações clínicas relacionadas à origem, tipos histológicos, fatores de risco, sítios anatômicos e respostas ao tratamento. Os tumores pediátricos na maioria das vezes são de origem embrionária e manifestam curto período de latência, portanto, os fatores ambientais exercem pouca ou nenhuma influência, somado a isso, possuem sintomatologia inespecífica e tendem a ser mais agressivos e com evolução mais rápida (Lima et al., 2018).

Os tipos de cânceres mais frequentes que acometem a população infantil são a leucemia, os tumores que atingem o sistema nervoso central, neuroblastoma, tumor de Wilms, linfomas e retinoblastoma, sendo que geralmente surgem durante a primeira infância. Enquanto nas crianças mais velhas e nos adolescentes, os tipos de cânceres que prevalecem são os tumores ósseos, mesmo podendo se desenvolver em qualquer idade. Já o osteossarcoma e o Tumor de Ewing são mais comuns em adolescentes. Outros tipos de cânceres são raros em crianças, mas podem acontecer. Em casos muito raros, as crianças podem até desenvolver cânceres que são mais comuns em adultos (American Cancer Society, 2019).

Por ano, mais de 200.000 crianças são diagnosticadas com câncer, compreendendo grande parte dos países em desenvolvimento como a segunda causa de mortalidade infantil após o primeiro ano de vida. No Brasil, o Instituto Nacional de Câncer (INCA) no ano de 2020, estimou que para cada ano do triênio 2020/2022, sejam diagnosticados 8.460 novos casos de câncer infanto-juvenis, sendo 4.310 homens e 4.150 mulheres. Esses valores correspondem a um risco estimado de 137,87 casos novos por milhão no sexo masculino e de 139,04 por milhão para o sexo feminino.

A melhoria do prognóstico nas últimas décadas permitiu que mais de $80 \%$ das crianças e adolescente diagnosticadas com câncer conseguissem sobreviver à doença (World Health Organization, 2017). Dentre às dificuldades vivenciadas por essas crianças e adolescentes em internações oncológicas, as mesmas não se resumem a dimensão biofisiológica da dor devido aos tratamentos, mas também, inclui o emocional, psicológico e social, resultado de um conjunto de sentimentos negativos e de difícil aceitação (Caires et al., 2018).

Diante desse cenário, a área de oncologia pediátrica necessita de melhorias constantes, no que diz respeito à prestação de uma assistência de qualidade para os usuários dos serviços de saúde, o enfrentamento do problema de forma integral, a 
adequação ao tratamento e suas complexidades, os reflexos perceptíveis ao nível familiar e social, devem ser trabalhados e enfrentados de forma atualizada, orientada, e embasada nos princípios éticos e científicos, de modo a proporcionar um cuidado eficaz e otimizado (Dias et al., 2020; Downing et al., 2018).

As capacitações e atualizações realizadas a nível profissional são de suma importância para a construção do conhecimento e desenvolvimento de ações práticas adequadas nas situações de tratamento, influenciando diretamente na aplicação de recursos e no atendimento integral à criança acometida pelo câncer. As capacitações também tornam o profissional mais seguro perante as atitudes e decisões a serem tomadas e propiciam alicerce para melhorar a relação e comunicação, entre paciente e profissional, profissional e familiar (Guimarães et al., 2017).

Apesar das inúmeras contribuições das capacitações para o exercício profissional, a atuação na instituição e o rompimento de barreiras relacionais percebem-se, no contexto atual, uma enorme lacuna no tocante a oferta de treinamento, principalmente na área de oncologia pediátrica. Essa realidade vem sendo evidenciada desde os cursos técnicos à graduação, os quais visam formar o profissional de enfermagem generalista, deixando de lado a oferta de cursos ou matérias direcionadas a essa área que necessita de um cuidado diferenciado e holístico, de acordo com as novas perspectivas sociais e de saúde pública (Amador et al., 2011).

Essa linha tênue de dificuldades de realização de capacitações também atinge significativamente o universo profissional, onde as instituições deixam em segundo plano à disposição das mesmas, tornando escassas as oportunidades e inexata à frequência de promoção o que pode prejudicar a qualidade da assistência prestada pela equipe de enfermagem (Silva et al., 2015).

A enfermagem tem um quantitativo expressivo de profissionais, que, segundo COFEN (2007), perpassa outras profissões atuantes na área de saúde. Os profissionais dessa área atuam diretamente com a criança com neoplasia e seus familiares, ao realizar diversos procedimentos inerentes ao tratamento, que muitas vezes geram dor, o enfermeiro necessita dispor de uma abordagem diferenciada, e para tal o entendimento do universo da criança torna-se essencial (Caires et al., 2018).

Desta forma identifica-se a importância da atualização profissional nos diferentes cenários de cuidado, principalmente no contexto do câncer infantil, de forma a conduzir o aprimoramento de competências, bem como a valorização dos saberes. A partir disso, surgiram os seguintes questionamentos: Os profissionais que atuam em unidades de oncologia pediátrica possuem capacitação específica na área? As instituições de saúde com atendimento oncológico capacitam suas equipes em prol de uma assistência qualificada?

Para sanar essas perguntas, propõe-se através desta pesquisa, identificar a frequência de capacitação da equipe de enfermagem de uma unidade oncológica pediátrica e a articulação dos conhecimentos adquiridos com a assistência prestada. Essa discussão torna-se relevante, pois, partindo do pressuposto que, a qualificação profissional é o caminho para o alcance de uma melhor qualidade da assistência integral à criança com diagnóstico de câncer, o trabalho poderá contribuir para reflexão dos profissionais e dos responsáveis pelas instituições de saúde.

\section{Metodologia}

Trata-se de um estudo de caso com abordagem do tipo exploratória, descritiva, de natureza quantitativa. Para Pereira et al. (2018) a coleta de dados trata-se de um método quantitativo e seu emprego objetiva realizar previsões de algum fenômeno em estudo na área da saúde, portanto possibilita uma busca aprofundada do objeto de análise, permitindo maior flexibilidade na condução da pesquisa.

Para elaborar a questão norteadora utilizou-se a estratégia PICO (População, Intervenção, Controle e Resultados), realizada de acordo com as seguintes etapas de elaboração: escolha do tema, produção da questão norteadora, busca na 
literatura, coleta de dados, seleção e análise crítica dos estudos, discussão dos resultados e apresentação da revisão.

O projeto foi submetido ao Comitê de Ética em Pesquisa da UFS - Plataforma Brasil e aprovado com CAE $n^{\circ}$ 58536516.1.0000.5546. Todos os participantes assinaram o Termo de Consentimento Livre e Esclarecido (TCLE) e os pesquisadores respeitaram os aspectos éticos de acordo com a Resolução 466/2012 do Conselho Nacional de Saúde (CNS).

Como instrumento de coleta foi aplicado um questionário embasado nas pesquisas de Mutti et al. (2016) intitulado como "Especialidade do ser profissional de enfermagem no mundo do cuidado à criança com câncer"; por Dias et al. (2013) intitulado como "Enfermeiro Clínico Especialista: um modelo de prática avançada de enfermagem em oncologia pediátrica no Brasil"; realizado por Erdmann, Fernandes e Teixeira (2016), intitulado como "Panorama da educação em enfermagem no Brasil: graduação e pós-graduação".

A coleta dos dados ocorreu no período de março e abril de 2017 em um hospital do Estado de Sergipe, cuja escolha se justifica por tratar-se de uma referência em Oncologia. Os participantes foram recrutados com base nos seguintes critérios de inclusão: fazer parte da equipe de enfermagem em nível superior ou técnico, alocados na unidade da pesquisa por período mínimo de um ano.

Após a seleção dos possíveis participantes, foi enviada uma carta convite, contendo informações, relevância e objetivos do estudo. Mediante a manifestação livre e esclarecida de concordância com os termos da pesquisa, o questionário foi aplicado, respondido e posteriormente organizado em banco de dados através do programa Microsoft Office Excel 2016. Com os dados organizados, aplicou-se a estatística simples para a análise quantitativa, em seguida os mesmos foram apresentados em porcentagens e convertidos em forma de gráficos e tabelas.

\section{Resultados e Discussão}

Considerando o perfil dos profissionais, foi verificado predomínio do sexo feminino. A média de idade dos participantes foi de 41 anos, com variação entre 30 e 40 anos. O tempo médio na profissão foi de 14 anos e de oito anos na profissão em oncologia pediátrica. Em relação às categorias profissionais de enfermagem, o grupo de maior representação foram o dos técnicos $(58,7 \%)$, seguidos dos auxiliares $(30,2 \%)$ e enfermeiros $(11,1 \%)$, como pode ser analisado na Figura 1(A). 
Figura 1 - Perfil dos entrevistados (A) Categoria profissional (B) sexo.

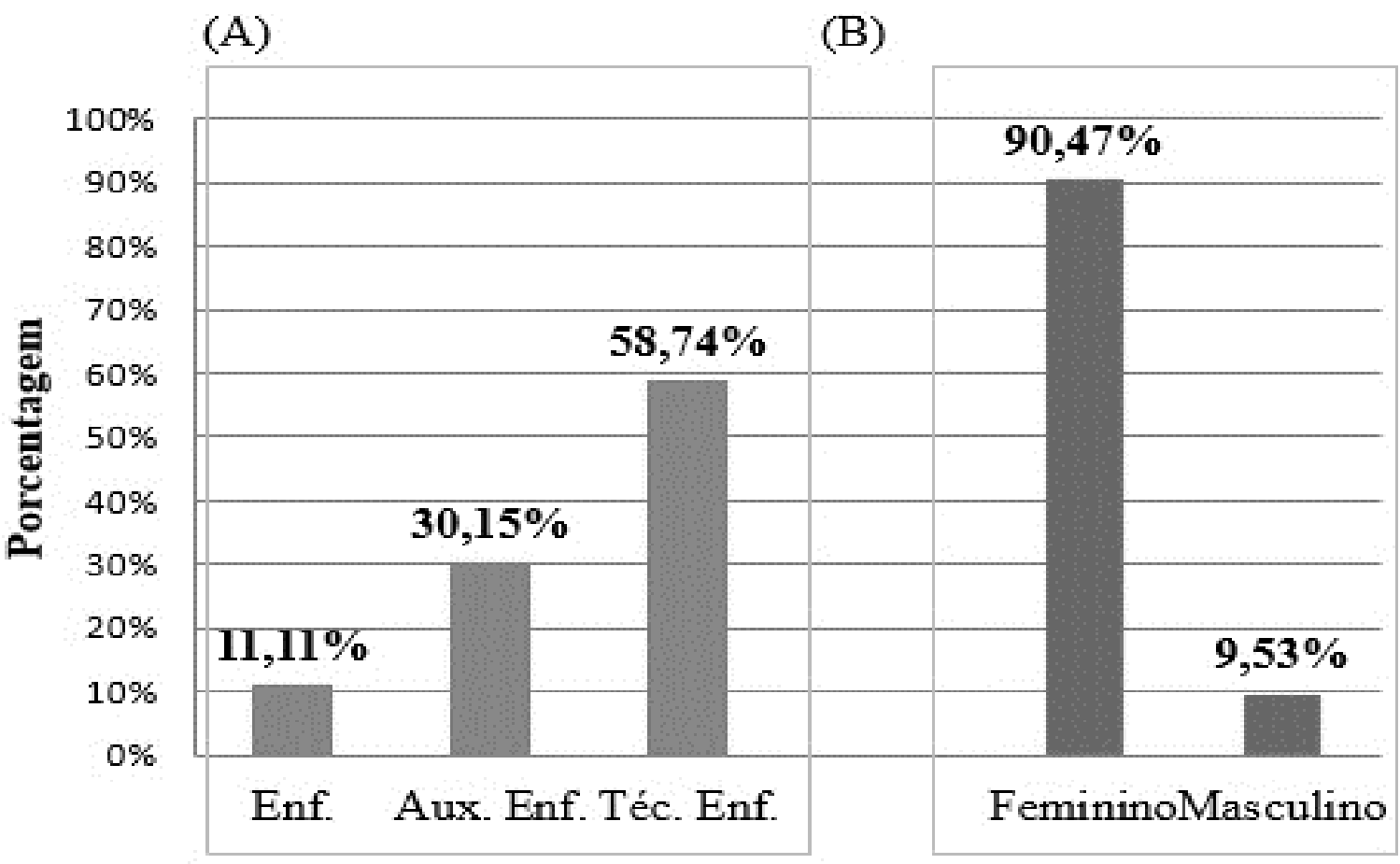

Fonte: Pesquisa (2017).

Portanto, os técnicos de enfermagem apresentam uma maior porcentagem de profissionais que atuam nesse serviço de saúde hospitalar, sendo que o curso técnico, por ter uma durabilidade inferior quando comparado ao nível superior, não abordam sobre a oncologia pediátrica, causando uma lacuna na formação dos profissionais, percebida na assistência prestada quando já se encontra formado.

Mediante dados obtidos a partir das respostas do questionário aplicado, conforme a Tabela 1 é nítida a relevância da temática, partindo do pressuposto de que mais da metade (58,7\%) dos profissionais não obtiveram nenhum tipo de conhecimento voltado para oncologia no seu processo de formação. Após o período de formação profissional, pouco mais da metade dos entrevistados, apenas 54\%, buscaram, de alguma forma, aprimorar seus conhecimentos na área da oncologia pediátrica. 
Tabela 1 - Capacitações dos profissionais de enfermagem na área de oncologia pediátrica. Aracaju - SE, Brasil, 2017.

\begin{tabular}{|c|c|c|c|}
\hline \multicolumn{2}{|l|}{ Variáveis } & \multirow{2}{*}{$\begin{array}{l}\mathbf{N}(\mathbf{n}=\mathbf{6 3}) \\
26\end{array}$} & \multirow{2}{*}{$\%$} \\
\hline Durante a formação profissional em enfermagem, cursou alguma & Sim & & \\
\hline & Não & 37 & $\mathbf{5 8 , 7}$ \\
\hline \multirow{2}{*}{$\begin{array}{l}\text { Após a formação profissional em enfermagem, participou de algum } \\
\text { curso de capacitação e/ou especialização em oncologia pediátrica? }\end{array}$} & Sim & 34 & 54 \\
\hline & Não & 29 & 46 \\
\hline \multirow{2}{*}{$\begin{array}{l}\mathrm{Na} \text { instituição de trabalho atual, já participou de cursos de atualização } \\
\text { em oncologia pediátrica? }\end{array}$} & Sim & 8 & 12,7 \\
\hline & Não & 55 & 87,3 \\
\hline \multirow{5}{*}{$\begin{array}{l}\text { As capacitações realizadas foram custeadas pela instituição hospitalar } \\
\text { ou através de recursos próprios? }\end{array}$} & $\mathrm{IH}$ & 29 & 46,03 \\
\hline & Não respondeu & 15 & 23,83 \\
\hline & IH e RP & 11 & 17,46 \\
\hline & $\mathrm{RP}$ & 4 & 6,34 \\
\hline & Não realizado & 4 & 6,34 \\
\hline \multirow[t]{7}{*}{ De que forma a capacitação influenciou na sua atuação profissional? } & AP & 24 & 38,09 \\
\hline & Atualização & 11 & 17,47 \\
\hline & Não respondeu & 11 & 17,47 \\
\hline & MAAP & 10 & 15,88 \\
\hline & MDP & 4 & 6,34 \\
\hline & Não influenciou & 2 & 3,17 \\
\hline & Prática correta & 1 & 1,58 \\
\hline
\end{tabular}

Legenda: IH - Instituição Hospitalar; RP - Recursos próprios; AP- Aprimoramento do Conhecimento; MAAP- Melhoria do Atendimento da Assistência Prestada: MDPP- Melhor Desenvolvimento na Prática. Fonte: Pesquisa (2017).

Quando questionados se na atual instituição de trabalho participaram de algum curso de atualização em oncologia pediátrica, 87,3\% dos profissionais entrevistados responderam que não. No que concerne aos custos das capacitações realizadas, $46 \%$ dos entrevistados referem que os treinamentos foram financiados pela instituição hospitalar, e aproximadamente $6 \%$ referiram custeios próprios.

Quando realizado o questionamento sobre os benefícios das capacitações, os profissionais relataram reflexos positivos, constataram-se, em ordem decrescente, os seguintes aspectos: aprimoramento do conhecimento e teorias, atualizações, melhoria no atendimento e assistência prestados, melhor desenvolvimento na prática profissional, realização de um trabalho correto para a proteção dos pacientes e profissionais, com mais segurança, além de maior qualificação profissional. A experiência profissional, juntamente com as qualificações irá determinar o perfil do enfermeiro.

Embora esses profissionais visualizem diversas vantagens, alguns empecilhos dificultam a adesão dessa educação continuada por meio de capacitações, refletindo diretamente na assistência prestada ao público estudado, conforme apresentado na Figura 2. 
Figura 2 - Dificuldades enfrentadas na capacitação profissional pela equipe de enfermagem na unidade hospitalar.

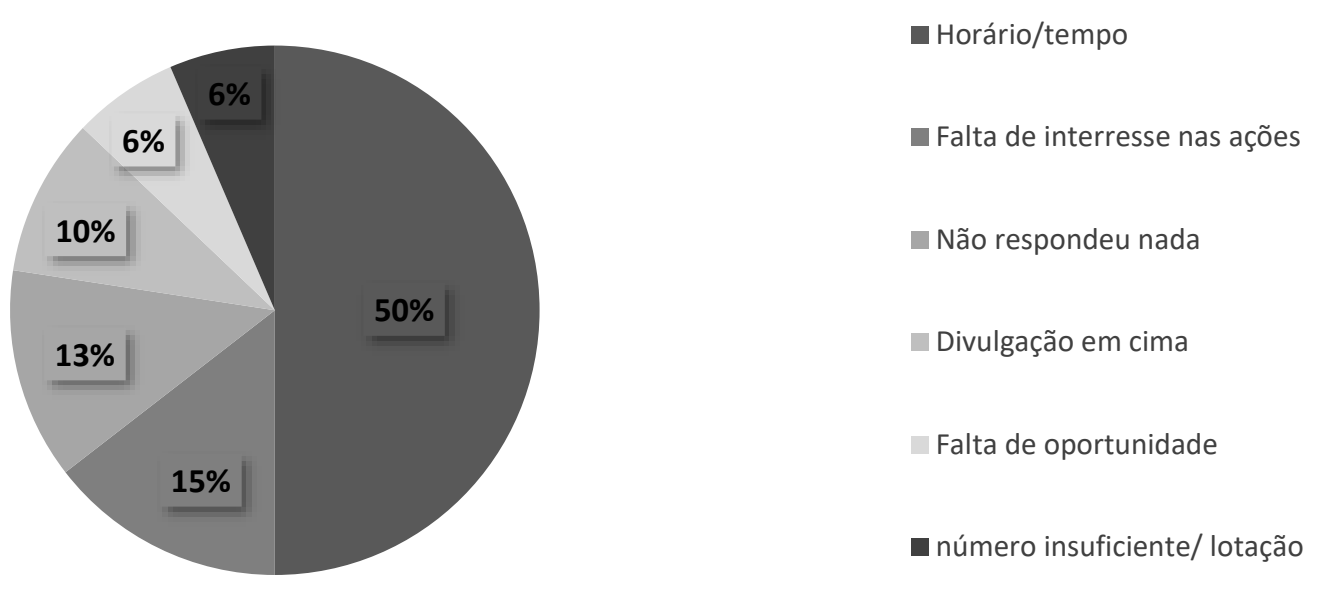

Fonte: Pesquisa (2017).

No que se refere às dificuldades enfrentadas na capacitação profissional pela equipe de enfermagem na unidade hospitalar, o fator que predomina com $31 \%$ é voltado ao horário cujos treinamentos são realizados e a falta de tempo para participar, a equipe relata ocorrer choque de horário com outras atividades e plantões de outras instituições, além de acusar divulgação em cima da hora, impossibilitando organização prévia. Entretanto, um dado que chamou bastante atenção foi que $15 \%$ dos entrevistados desacreditam nas ações de aperfeiçoamento profissional (Figura 2).

Visto benefícios e empecilhos expostos pela equipe de enfermagem, foi então indagada se a instituição hospitalar incentiva os profissionais a se capacitarem, a partir desse questionamento foram obtidos os seguintes resultados: $61,9 \%$ dos profissionais responderam que não e $38,1 \%$ responderam que sim, para estes foi explorado qual forma de incentivo, como mostra na Figura 3.

Figura 3 - Forma de incentivo à capacitação dos profissionais pela instituição hospitalar.

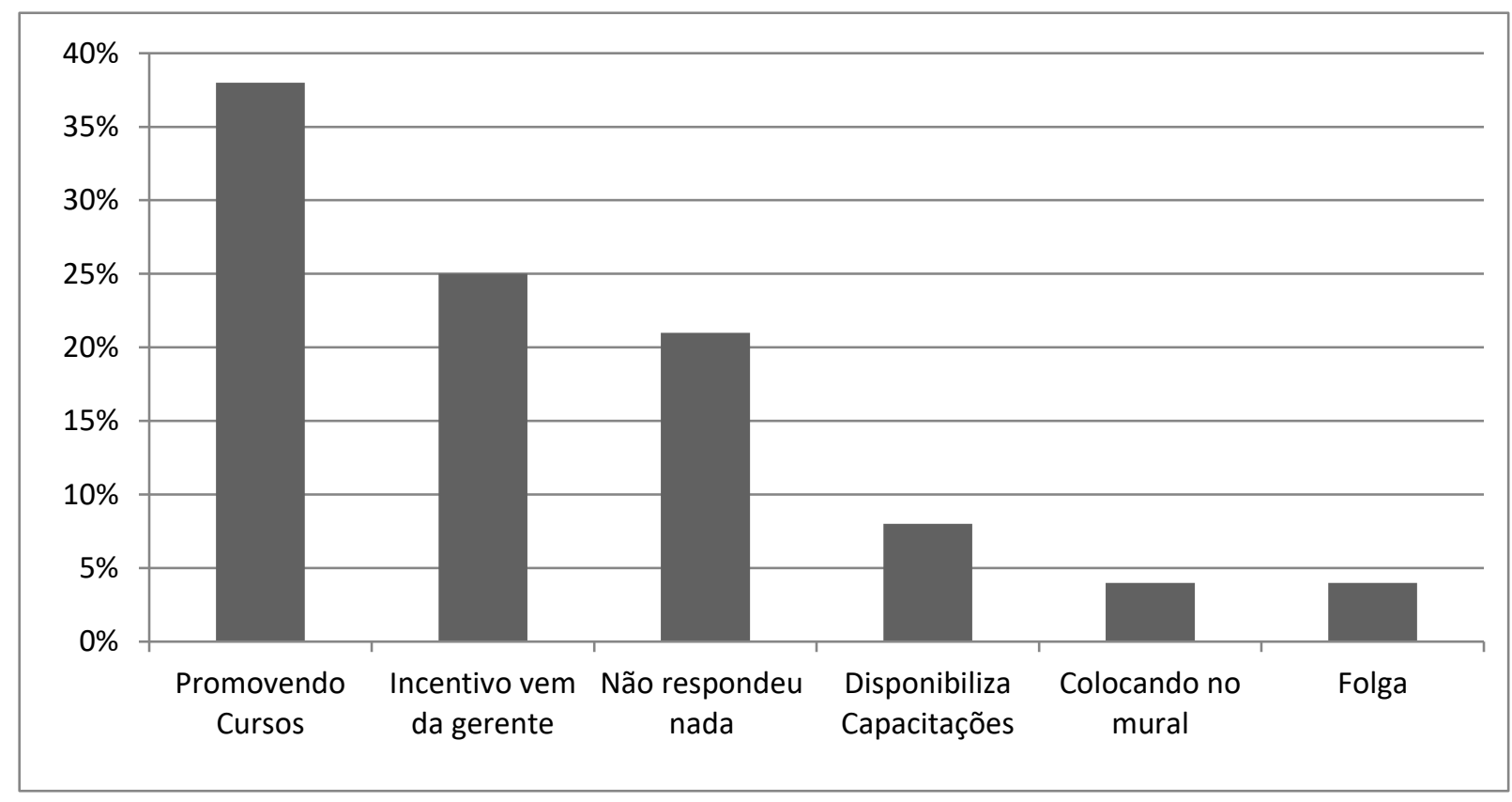

Fonte: Pesquisa (2017). 
Diante disso, a realização de cursos voltados à oncologia pediátrica demonstrou ser a forma de incentivo que despertou maior interesse entre os profissionais. Em seguida, 25\% dos profissionais relataram que os incentivos partem da gerência. Posteriormente, a disponibilização de capacitações fixadas no mural, assim como às folgas foram consideradas formas de incentivo. No entanto, mais de $20 \%$ dos profissionais entrevistados não expressaram formas de incentivo (Figura 3 ).

Em referência à oncologia, notadamente à oncologia pediátrica, são temas pouco abordados durante o processo de formação, tendo em vista que os cursos de graduação em enfermagem têm como foco a formação de um enfermeiro generalista. E após o período de formação profissional, pouco mais da metade dos entrevistados, buscaram, de alguma forma, aprimorar seus conhecimentos na área da oncologia pediátrica. Esses números mostraram-se insuficientes, dessa forma, foram equiparados aos estudos de Dias, de Da Silva e de Reichert, os quais abordam que o ideal é que todos os profissionais busquem capacitar-se.

A busca pelo conhecimento permite uma reflexão e a criticidade que auxilia para o perfil do enfermeiro que a sociedade atual: com princípios morais, autoconfiante e que assegure uma assistência de enfermagem qualificada. Cabe ao profissional reconhecer os benefícios e a importância de buscar esse conhecimento em áreas tão peculiares, tanto para seu crescimento profissional, quanto para assistência adequada ao paciente (Dias et al., 2013).

$\mathrm{Na}$ enfermagem, o trabalho em equipe requer a interdependência das ações de profissionais de nível técnico e superior que, além de compartilharem a técnica do trabalho, propiciem relações humanas satisfatórias para a realização de uma assistência segura, eficiente e eficaz (Souza et al., 2015).

Quando questionados se na atual instituição de trabalho participou de algum curso de atualização em oncologia pediátrica, ficou visível que ocorre um descompasso do que se é idealizado, pois prestar assistência à criança com câncer é um grande desafio e requer, além da estrutura, conhecimento especifico. Nesta perspectiva, as capacitações são estratégias utilizadas para melhoria da atuação profissional, possibilitando o pensar crítico reflexivo quanto às práticas realizadas (Galdino, Silva \& Elias, 2016).

No que concerne aos custos das capacitações realizadas, foi exposto que maior parte dos profissionais realizou capacitações custeadas por meio da instituição hospitalar, e um número muito pequeno referiram realizar por meio de recursos próprios. Visando atender a uma demanda de consolidação do SUS, através da transformação do profissional em sujeito, em fevereiro de 2004 foi instituída a Política Nacional de Educação Permanente, através da Portaria 198/GM/MS, com a finalidade de formar e capacitar profissionais da saúde para atenderem às reais necessidades da população.

Por isso, a educação serve, então, como proposta de mudança dos serviços de saúde, contínua, válida e atual, motivando a ação de trabalhadores, gestores e usuários do SUS (Ministério da Saúde, 2018). No entanto, a busca pelo conhecimento deve também acontecer por parte dos profissionais de forma crescente e evolutiva, incumbindo, não somente o desenvolvimento intelectual de cada profissional, mas na própria profissão, em sua trajetória histórica e na construção de saberes (Souza et al., 2020).

Ao analisar os resultados, $15 \%$ dos profissionais relatam não visualizar benefícios nas capacitações. Esses agem de forma oposta ao que preconiza o código de ética dos profissionais de enfermagem, resolução COFEN 311/2007, no qual consta que é direito (artigo $2^{\circ}$ ), mas também é dever (artigo $14^{\circ}$ ), que compete ao profissional de forma individual ou coletiva, estar atento as melhorias que a capacitação trará: aperfeiçoamento e qualidade da assistência prestada, aprimorando seus conhecimentos como alicerce a sua prática profissional. Essas capacitações são indispensáveis para o mundo atual repleto de velozes mudanças científicas e tecnológicas (COFEN, 2007).

Em relação ao incentivo às capacitações, dado pela instituição hospitalar, ficou visível que a maioria dos entrevistados, 61,9\%, sente-se desmotivados a capacitar-se. Diante disso, identificou-se uma divergência nas respostas obtidas 
com o que se preconiza na Portaria $\mathrm{n}^{\circ} 278$, em que o aprender e o ensinar devem ser introduzidos na rotina do profissional de enfermagem, assim como da instituição de trabalho.

As necessidades de capacitação encontram-se alicerçadas na problematização do processo e da qualidade do trabalho, em que o serviço prestado assegura a execução e a relevância dos conteúdos estabelecidos, que no caso da equipe, seria a pediatria. Cabe salientar que para isso é de suma importância incentivar os profissionais para a busca e realização das capacitações, visando à melhoria da assistência prestada e a resolução otimizada dos problemas identificados (Galdino, Silva \& Elias, 2016).

\section{Considerações Finais}

A carência em treinamento e capacitações direcionadas à oncologia pediátrica é nítida, demonstrando um grande obstáculo à melhoria da assistência que compete à equipe de enfermagem. Essa deficiência de promoção de capacitações ainda é visível na formação profissional, e concomitantemente atinge o universo da prática de trabalho. Em relação ao enfermeiro, denota-se a necessidade de modificações curriculares que busquem atender às exigências do mercado de trabalho, favorecendo o desenvolvimento de competências e de habilidades gerais e específicas e, por consequência de um profissional qualificado, reflexivo e pronto para atuar sobre a realidade epidemiológica e social na área oncológica.

Portanto, as contribuições dessa pesquisa remetem a importância do papel das instituições de ensino na formação dos futuros profissionais de enfermagem, sugerindo que essa temática oncologia pediátrica seja introduzida com mais ênfase durante os cursos técnicos e de nível superior. Além disso, busca trazer um despertamento das instituições de trabalho acerca da importância da realização de capacitações sobre essa área tão peculiar. Diante disso, espera-se proporcionar uma reflexão do profissional quanto ao seu papel assistencial e conscientizá-lo da relevância de buscar o conhecimento de forma contínua, assim como fortalecer o desenvolvimento da pesquisa na área em questão.

Nota-se a relevância deste artigo como estratégia para buscar dados atualizados relacionados à capacitação profissional frente às crianças com cânceres. Com isso, recomenda-se a ampliação de pesquisas futuras referentes à temática visando agregar novas evidências que enfatizem a importância desses treinamentos com os profissionais da área de enfermagem e práticas com estudantes ainda na graduação, para que seja possível fornecer uma assistência qualificada e que atenda à demanda desses jovens.

\section{Referências}

Amador, D. D., Gomes, I. P., Coutinho, S. E. D., Costa, T. N. A., \& Collet, N. (2011). Concepção dos enfermeiros acerca da capacitação no cuidado à criança com câncer. Texto \& Contexto - enfermagem, 20(1), 94-101.

American Cancer Society (2019). Cancer in children: types of cancer that develop in children https://www.cancer.org/cancer/cancer-in-children/types-ofchildhood-cancers.html

Caires, S., Machado, M., Antunes, M. C., \& Melo, A. S. M. (2018). Recidiva oncológica: olhares dos profissionais hospitalares sobre as dificuldades do paciente pediátrico. Psico-USF, 23(2), 333-345.

Conselho federal de enfermagem (COFEN). Resolução COFEN nº 311/2007 Código de Ética dos Profissionais de Enfermagem. 2007. http://www.cofen.gov.br/wp-content/uploads/2012/03/resolucao_311_anexo.pdf.

Dias, C. G., Duarte, A. M., Ibanez, A. S. S., Rodrigues, D, B., Barros, D. P., Soares, J. S., Perlin, J. P. M., Santos, N. S., Paiva, P. M., \& Gutiérrez, M. G. R. (2013). Enfermeiro Clínico Especialista: um modelo de prática avançada de enfermagem em oncologia pediátrica no Brasil. Rev. esc. enferm. USP 47(6), 1426-1430.

Dias, K. C. C. O., Batista, P. S. S., Fernandes, M. A., Zaccara, A. A. L., Oliveira, T. C., Vasconcelos, M. F., Oliveira, A. M. M., \& Andrade, F. F. (2020). Dissertações e teses sobre cuidados paliativos em oncologia pediátrica: estudo bibliométrico. Actapaul. enferm., $33,1-8$.

Downing, J., Boucher, S., Daniels, A., \& Nkosi, B. (2018). Pediatric palliative care in resource-poor countries. Children (Basel). 5(2), 1-12.

Erdmann, A. L., Fernandes, J. D., \& Teixeira, G. A. (2011). Panorama da educação em enfermagem no Brasil: graduação e pós-graduação. Enfermagem em Foco, 2, 89-93. 
Research, Society and Development, v. 10, n. 6, e4710615475, 2021

(CC BY 4.0) | ISSN 2525-3409 | DOI: http://dx.doi.org/10.33448/rsd-v10i6.15475

Feliciano, S. V. M., de Oliveira Santos, M., \& Pombo-de-Oliveira, M. S. (2018). Incidência e mortalidade por câncer entre crianças e adolescentes: uma revisão narrativa. Revista Brasileira de Cancerologia, 64(3), 389-396.

Galdino, J. P. S., Silva, E. T., \& Elias, F. T. S. (2016). Oportunidades de formação em avaliação de tecnologias em saúde: mapeamento de cursos nacionais e internacionais. Com. Ciências Saúde, 27(3), 203-210.

Guimaraes, T. M., Silva, L. F., Santo, F. H. E., Moraes, J. R. M. M., \& Pacheco, S. T. A. (2017). Cuidado paliativo em oncologia pediátrica na formação do enfermeiro. Rev. Gaúcha Enferm., 38(1), 1-9.

Instituto Oncoguia (2020). Estatísticas para Câncer Infantil http://www.oncoguia.org.br/conteudo/estatisticas-para-cancerinfantil/10665/459/\#: :text=O\%20Instituto\%20Nacional\%20de\%20C\%C3\%A2ncer,homens\%20e\%204.150\%20em\%20mulheres

Lima, B. C., Silva, L. F., Góes, F. G. B., Ribeiro, Martha T. S., \& Alves, L. L. (2018). O itinerário terapêutico de famílias de crianças com câncer: dificuldades encontradas neste percurso. Revista Gaúcha de Enfermagem, 39(e2018004), 1-9.

Ministério da Saúde (2004). Portaria no 198/GM/MS, de 13 de fevereiro de 2004. Brasília, Ministério da saúde, 141(32), 37-41.

Ministério da Saúde. (2018). Política Nacional de Educação Permanente em Saúde: o que se tem produzido para o seu fortalecimento? Ministério da Saúde, Secretaria de Gestão do Trabalho e da Educação na Saúde, Departamento de Gestão da Educação na Saúde. Brasília, DF: Ministério da Saúde, 9-10.

Mutti, C. F., Padoin, S. M. M., \& Paula, C. C. (2012). Espacialidade do ser profissional de enfermagem no mundo do cuidado à criança que tem câncer. Esc Anna Nery(impr.), 16 (3),493-499.

Pereira, A. S. et al. (2018). Metodologia da pesquisa científica. UFSM.

Silva, A. F., Issi, H. B., Motta, M. G. C., \& Bonete, D. Z. A. (2015). Palliative care in pediatric oncology: perceptions, expertise and practices from the perspective of the multidisciplinary team. Rev Gaúcha Enferm, 36(2), 56-62.

Souza, I. A. S., Pereira, M. O., Oliveira, M. A. F., Pinho, P. H., \& Gonçalves, M. D. A. (2015). Work process and its impact on mental health nursing professionals. Acta Paul Enferm, 28(5), 447-453.

Souza, R. S., Araújo, F. L. de, Manzo, B. F., Marcatto, J. O., Montenegro, L. C., Silva, P. R. M., \& Simão, D. A. S. (2020). O cuidado na oncologia pediátrica: análise transversal da qualidade de vida de profissionais de enfermagem. Revista Brasileira de Enfermagem, 73(6), 1-8.

World Health Organization (2017). Cancer https://www.who.int/health-topics/cancer\#tab=tab_1 